growth: thermodynamics of phase equilibria, kinetics of nucleation and growth (this latter restricted to the bare minimum, as it should be in this type of book), morphological characteristics, altogether condensed into 68 pages. Then follow chapters on the various methods of crystal growth: from the gas phase (168 pp.), from solution (70 pp.), hydrothermal growth (41 pp.), from fluxes (99 pp.), from the melt (277 pp.) and from the solid state (12 pp.). Thereafter some special methods are treated: growth under conditions of high pressure, high and low temperature, and growth of dendrites, whiskers and polymers. The next chapter deals with all types of defects that grown crystals may have, while the last chapter contains a survey of practical problems: materials for ampoules and crucibles, heating methods, temperature measurement, preparations of pure substances and where to get commercially available crystal growth equipment.

Every method is introduced by a general and theoretical treatment, just enough to understand what exactly one is doing. Then the equipment is described in an extremely clear and detailed manner, while several hints for appropriate manipulation are given. Substances that have been grown by the method under discussion are put together in extensive tables (often 20 pages or more) with details about the methods and the results. Other tables follow covering investigations carried out with the grown crystals on special topics such as condensation coefficients, dislocation structures, morphology, growth rates, etching, nucleation, polymorphism and impurity contents.

Sometimes the theory is incorporated in a different chapter, for example the zone-melting theory is treated in the chapter on the defect structures. Extensive indexes of subjects and substances, the latter with about 1700 entries, greatly facilitate the search for information. The literature is reviewed up to 1970 and the 4000 references have been assembled in a separate booklet.

In general the text is very clear and the illustrations have been very carefully designed, although the photographs sometimes lack the clarity they need, owing to the quality of the paper. Very few errors have been found; their number must be of the same order of magnitude as the number of defects in the purest dislocation-free crystal ever grown.

Anyone who has to grow crystals will feel indebted to K.-Th. Wilke, who, assisted by J. Bohm, undertook the tre- mendous work of preparing this compilation. This book is a must for the library of every crystal growth laboratory.

\section{P. HARTMAN}

Geologisch en Mineralogisch Instituut
$\quad$ der Rijksuniversiteit
Garenmarkt $1 B$
Leiden
Netherlands

Titanium - science \& technology, Vols. 1, 2, 3, and 4. Edited by R. I. Jaffee and H. M. BuRte. Vol. 1: Pp.xxvii+767, Figs. 346. Tables 98. Vol. 2: Pp. 656, Figs. 304, Tables 75. Vol: 3. Pp. 689, Figs. 326, Tables 84. Vol. 4: Pp. 613, Figs. 242, Tables 76. New York: Plenum Press, 1973. Price $\$ 132.50$ per 4 volumes.

The Second International Conference on Titanium was held at the Massachusetts Institute of Technology in May 1972, four years after the inaugural conference in London. It was concerned to relate science and practical application, and covered the spectrum from fundamental research to service experience: it ranged through extraction and fabrication metallurgy to structure, properties and structure/property relationships. Major contributions were made from the countries of the sponsoring organisations - the U.S.A., the USSR, Japan and Great Britain. The presentation of the research papers (totalling nearly 200) involved a rapporteur system, and critical reviews of the major fields were also presented. The conference proceedings include the full text of the papers and reviews and also brief reports of the discussions. The format of the proceedings involves reproduction of typed manuscripts, with a range of type styles. Diagrams and photographs are clearly reproduced and there is a name index and a subject index. The adoption of a single system of units throughout the volumes was not a feature of the conference organization.

In reviewing such an extensive compilation of papers it is not possible to deal critically and in detail with the individual contributions, but rather it is appropriate to survey the overall contents of the volumes. Concerning the balance between the main fields of study, a large part of the conference dealt with structure/property relation- ships, with the control of structure and properties through processing, and the relation to service applications. In these areas there is much to interest the materials scientist, physical metallurgist and solid-state physicist, including detailed structural investigations, for example, using electron microscopy. The key to the control of many properties lies in the understanding of the phase transformations derived from the $\alpha / \beta$ allotropic change in titanium. Developments in this complex area have been substantial and it is interesting to note the considerable effort devoted to the study of the best established of commercial alloys, i.e. $\mathrm{Ti}-6 \mathrm{Al}-4 \mathrm{~V}$, as well as to the newer alloys such as those of the $\beta$-type.

Section I of the proceedings is entitled Introductory Papers and Section II Current Uses and Future Possibilities, including Economics and Materials Policy. In this latter section the review shows the importance of economic aspects and in the papers, various fields of application, with specific examples, are considered, e.g. in the aircraft and automobile industries. Section III, entitled Winning and Refining includes a review of technological developments since 1965, and refers to the increasing need to use sources of lower quality than rutile. The research papers deal with various extraction procedures, powder production, interaction with refractory oxides and scrap reclamation. Section IV, Consolidation. Primary and Secondary Fabrication, contains a review of developments in the U.S.A. and papers dealing with melting, casting, powder metallurgy, isostatic pressing, extrusion, forging and machining. In Section V, Joining, Quality Assurance and Inspection, the review and papers are concerned with a range of welding and inspection topics, such as electronbeam welding, diffusion bonding and ultrasonic inspection.

In Vol. 2, Section VI, Physical Properties, Electronic Structure, Phase Stability and Phase Equilibria, the review paper deals with calculations of phase stability and solubility limits in binary and ternary systems. Other papers in this section are concerned with electronic and mechanical properties and structure/ property relationships (e.g., superconductivity); elastic properties; internal friction in $\alpha$ and $\beta$ alloys; lattice defects, electronic structure and diffusion mobility; interaction of oxygen and hydrogen in titanium; thermodynamics of $\alpha$ stabilized $\mathrm{Ti}-\mathrm{X}-\mathrm{Y}$ systems and phase equilibria in certain titanium alloys. 
Section VII, Deformation and Fracture forms the greatest part of Volume 2. The comprehensive review paper makes particular reference to controlling mechanisms. Aspects of deformation in a wide range of alloys covered by research papers include the stability of dislocations in hexagonal crystals; the tensile stress-strain curves of cold-worked titanium; grain size effects; deformation of $\mathrm{Ti}-\mathrm{Al}$ alloys, including the effects of $\mathrm{Ti}_{3} \mathrm{Al}$ precipitates; deformation of $\mathrm{Ti}_{3} \mathrm{Sn}$; asymmetry of slip in $\beta$ Ti-V crystals and the ductile-brittle transition in $\beta$ alloys; thermally activated deformation in various alloys; superplasticity; twinning effects; texture development. Fatigue and fracture form the subject of a number of papers; the research on fatigue includes mechanisms, and the effects of variable loads, of anisotropy and of section size; work on fracture is concerned particularly with the effects of texture and microstructure on fracture characteristics.

In Vol, 3 the subject of Section VIII, Kinetics and Phase Transformations, is of fundamental importance in the control and 'tailoring' of alloy properties. It is covered by a comprehensive review and by detailed papers on $\beta$-phase decomposition concerned with the formation of martensite and of $\omega$ (including morphology and structure/property relationships, reversion phenomena and dislocation configurations). Among the alloys studied were $\mathrm{Ti}-\mathrm{Zr}, \mathrm{Ti}-\mathrm{Zr}-\mathrm{O}$, Beta III, Ti-Mo, Ti-V, Ti-Al; grain growth kinetics in $\mathrm{Ti}-\mathrm{N}$ alloys are also discussed. Section IX, Metallurgical Synthesis draws attention to the 'synthesis' viewpoint for alloy development (i.e. the 'designing' of alloys to achieve desired structure and properties) and this concept is elaborated in the discussion. The research papers cover both the analysis and the synthesis viewpoints. There is a paper on the use of computer analysis for correlating microstructure and mechanical properties. Several papers deal with structure/property relationships including fracture toughness with special reference to $\alpha / \beta$ alloys, $e, g$. Ti-6Al-4V. Both heat treatment and processing variables (e.g., in forging and extrusion) are dealt with. The theme of thermomechanical treatment is pursued in a group of papers on $\beta$ alloys such as Beta III.

Section $X$ is entitled Low to Intermediate Temperature Alloys. The review briefly surveys the various alloy types: $\alpha, \alpha$-age-hardening, $\alpha-\beta$, metastable $\beta$. Most of the papers deal with the structure and properties of various alloys, e.g., the development of deep hardenable alloys, and the fatigue behaviour of the $\mathrm{Ti}-6 \mathrm{Al}-4 \mathrm{~V}$ alloy. There is also a paper on dendrite morphology and microsegregation.

In Volume 4, Section XI deals with High Temperature Alloys. The review surveys fundamental aspects of hightemperature deformation and future possibilities for overcoming the limitations of titanium alloys for high-temperature use. Research papers relate mainly to high-temperature mechanical properties of a range of alloys, including both $\alpha$-types and $\beta$-types, and cover both fundamental and applied consider. ations.

Section XII, Titanium Matrix Composites, does not contain a review paper. The topics covered in the research papers include compatible alloys, interfacial reactions, explosion bonded cladding on steels, and mechanical properties; fibre materials referred to include boron, graphite and alumina.

General Corrosion and Oxidation. Coatings is the title of Section XIII.
In the review there is special reference to aspects relevant to industrial applications. The papers represent studies of industrially significant problems of corrosion and oxidation, relating to a wide range of corrosive conditions and involving various mechanisms; several of the papers deal with surface coatings, including those used to resist wear.

In the concluding Section XIV, Stress Corrosion and Hydrogen Embrittlement, the extensive and detailed review covers three areas: applications, data generation and theoretical aspects. As in Section XIII the papers cover a range of industrially important problems and conditions.

Although the theme of the conference proceedings is a particular metal and its alloys, the range of subjects that are involved, both fundamental and applied, should attract a wide range of readers. It is unfortunate that the inevitably high price of the volumes is likely to limit the extent of their availability; even some libraries, whose users should have ready access to the volumes may find it difficult to meet the cost. However, for scientists and technologists with a specialized interest in titanium, these volumes form an essential source of reference. Also, those approaching from a more general standpoint are likely to find, (particularly in the critical reviews) their interest stimulated in the titaniumbased group of materials for which there appears to be much future potential.

D. R. F. WEST

Department of Metallurgy

and Materials Science

Royal School of Mines

Imperial College of Science

and Technology

Prince Consort Road

London SW7 2 BP

England 